\title{
A novel biomarker TERTmRNA is applicable for early detection of hepatoma
}

\author{
Norimasa Miura*1, Yukio Osaki2, Miki Nagashima³, Michimori Kohno ${ }^{4}$, Kensho Yorozu5, Kohei Shomori6, \\ Takamasa Kanbe7, Kenji Oyama5, Yukihiro Kishimoto7, Shigeo Maruyama5, Eijiro Noma8, Yutaka Horie5, \\ Masatoshi Kudo33, Seigo Sakaguchi8, Yasuaki Hirooka9 ${ }^{9}$, Hisao Ito6 , Hironaka Kawasaki, Junichi Hasegawa' and \\ Goshi Shiota10
}

\begin{abstract}
Backgrounds: We previously reported a highly sensitive method for serum human telomerase reverse transcriptase (hTERT) mRNA for hepatocellular carcinoma (HCC). a-fetoprotein (AFP) and des- $\gamma$-carboxy prothrombin (DCP) are good markers for HCC. In this study, we verified the significance of hTERTmRNA in a large scale multi-centered trial, collating quantified values with clinical course.
\end{abstract}

Methods: In 638 subjects including 303 patients with HCC, 89 with chronic hepatitis (CH), 45 with liver cirrhosis (LC) and 201 healthy individuals, we quantified serum hTERTmRNA using the real-time RT-PCR. We examined its sensitivity and specificity in HCC diagnosis, clinical significance, ROC curve analysis in comparison with other tumor markers, and its correlations with the clinical parameters using Pearson relative test and multivariate analyses. Furthermore, we performed a prospective and comparative study to observe the change of biomarkers, including hTERTmRNA in HCC patients receiving anti-cancer therapies.

Results: hTERTmRNA was demonstrated to be independently correlated with clinical parameters; tumor size and tumor differentiation ( $P<0.001$, each). The sensitivity/specificity of hTERTmRNA in HCC diagnosis showed 90.2\%/85.4\% for hTERT. hTERTmRNA proved to be superior to AFP, AFP-L3, and DCP in the diagnosis and underwent an indisputable change in response to therapy. The detection rate of small HCC by hTERTmRNA was superior to the other markers.

Conclusions: hTERTmRNA is superior to conventional tumor markers in the diagnosis and recurrence of HCC at an early stage.

\section{Background}

Since the discovery of circulating nucleic acids (CNAs) in plasma in 1948, many diagnostic applications have emerged. Recently, CNAs instead of a protein has appeared on this scene of practical diagnostic assay, suggesting that cell-free CNAs in the plasma/serum of cancer patients have characteristics of tumor-derived nucleic acids. In addition to DNA-derived from tumor cells [1-4], a recent development in this new field is the finding of tumor-related RNA in the plasma/serum of cancer patients [5]. These features include tyrosine kinase

* Correspondence: mnmiura@hotmail.com

1 Division of Pharmacotherapeutics, Department of Pathophysiological and Therapeutic Science, Faculty of Medicine, Tottori University, 86 Nishicho, Yonago, Tottori 683-8503, Japan

Full list of author information is available at the end of the article
mRNA [6], telomerase components [7,8], the mRNAs that are encoded by different tumor-related genes [9-13], and viral mRNA [14]. In one study, two telomerase markers in breast cancer yielded $44 \%$ of positive rates [7]. Nevertheless, telomerase RNA seems to be a promising marker by the reason that it can be found even in the serum of patients with small, undifferentiated breast cancers without any metastatic lesions. Dasi et al. showed that circulating telomerase RNA is a sensitive marker, using real-time reverse transcription-PCR (RT-PCR) [8].

The telomerase catalytic subunit (hTERT) exerts important cellular functions, including telomere homeostasis, genetic stability, cell survival and perhaps differentiation [15-20]. hTERTmRNA in serum was detected in 
breast cancer but not in benign diseases, suggesting that hTERT is available for cancer diagnosis [4].

HCC ranks high among the most common and fatal malignancies associated with hepatitis $B$ virus (HBV) and hepatitis $\mathrm{C}$ virus (HCV) infection [5]. Although HCC patients receive possible medical treatments such as transcatheter arterial chemoembolization/embolization (TACE/TAE), radiofrequency ablation (RFA), and surgery for primary tumors, intrahepatic and extrahepatic recurrence frequently limit patient's survival [6]. Although the modalities such as ultrasonography (US) and conventional tumor markers such as $\alpha$-fetoprotein-L3 (AFP-L3) and DCP are widely used and important for HCC detection in clinical scenes [7], they still do not provide an entirely satisfactory solution to detect HCC at the early stage. Since HCC has been recently classified as a complex disease with a wide range of risk factors and many cellular signaling pathways have been reported to be involved in hepatocarcinogenesis, a novel biomarker for $\mathrm{HCC}$ is required [21]. We previously reported that measurement of serum hTERTmRNA by real-time RT-PCR method was sensitive in detection of tumor-derived hTERTmRNA even in the HCC patients whose AFP levels were low [9], and was also useful even for other malignancies such as non-small cell lung cancer, ovarian cancer, and gastric cancer [22-24]. In this large-scale study that includes follow-up cases, we focused on HCC of all malignancies and assessed the clinical significance of hTERTmRNA measurement in HCC diagnosis and monitored the clinical course.

\section{Methods}

\section{Patients and Sample Collection}

Four hundred-thirty seven consecutive patients (303 patients with $\mathrm{HCC}, 89$ with $\mathrm{CH}$, and 45 with $\mathrm{LC}$ ), who were admitted at Tottori University related Hospitals, Osaka Red Cross Hospital, and Fukuoka University Chikushi Hospital between November, 2002 and December, 2006, were enrolled in this study. All the HCC patients had $\mathrm{LC}$ or $\mathrm{CH}$ as the underlying liver disease. The mean ages of patients with $\mathrm{HCC}$, LC, and $\mathrm{CH}$ were 65, 66, and 61 years, respectively. One hundred-sixty seven patients were infected with $\mathrm{HCV}, 97$ with $\mathrm{HBV}, 24$ with both viruses and 15 with no viral markers. The patients were diagnosed by blood chemistry, US, computed tomography (CT), AFP and/or biopsy under US. The clinicopathological findings (age, gender, etiology, underlying liver disease (adjacent lesion), Pugh score, Child classification, total bilirubin (TB), albumin (Alb), alanine aminotransferase (ALT), AFP, AFP-L3, DCP, HCV titer, HCV subtype, tumor number, tumor size, differentiation degree of tumor, and presence of metastasis) were evaluated (Figure 1). HCC was diagnosed according to the AASLD guidelines and the differentiation of $\mathrm{HCC}$ was diagnosed by liver biopsy. Two hundred one healthy individuals including 144 females (from 24-87 years old: mean age 57 years) served as controls. Informed consent was obtained from each patient and the study protocols followed the ethical guidelines of the 1975 Declaration of Helsinki and were approved by the human research committee of Tottori University. The therapies for HCC include TAE, transcatheter arterial infusion (TAI), percutaneus ethanol injection therapy (PEIT), and RFA. Regarding follow-up patients, blood samples were taken basically every two months.

\section{RNA extraction and Real-time quantitative RT-PCR}

Harvesting serum samples were performed as previously described [9]. RNA was extracted with DNase treatment from serum as reported previously [4,9]. The quantitative RT-PCR was performed as described previously $[5,10]$. (a) for hTERT. The RT-PCR condition was an initial incubation at 50 for $30 \mathrm{~min}$ followed by a 12-min incubation at 95 , then 50 cycles at $95(0 \mathrm{~s}), 55(10 \mathrm{~s})$, and $72(15 \mathrm{~s})$, and a 20 second melting at $40^{\circ} \mathrm{C}$. The dynamic ranges of real-time PCR analysis for hTERTmRNA were more than approximately 5 copies in this assay and we were able to exclude the possibility of false negativity in serum samples from patients with $\mathrm{CH}, \mathrm{LC}$ and controls. The PCR yielded products of $143 \mathrm{bp}$ for hTERT (data not shown). The RT-PCR assay was repeated twice and the quantification was confirmed by using LightCycler (Roche, Basel, Switzerland) with reproducibility.

\section{hTERTmRNA during the treatment and detection of small HCC}

We examined the therapeutic effectiveness of hTERTmRNA during the clinical course. Serum hTERTmRNA was measured before and 7 days after TAE in $16 \mathrm{HCC}$ patients. In comparison with AFPmRNA, the half-life of hTERTmRNA was examined. By monitoring gene expression in serum up to 6 months after the beginning of therapy such as TAE, TAI, RFA, PEIT, surgical treatment, the effect of therapies were estimated in 20 patients. Furthermore, we examined hTERTmRNA expression and level of other conventional tumor markers after they were categorized by the tumor size (less than $10 \mathrm{~mm}, 11-20 \mathrm{~mm}$, 21-30 $\mathrm{mm}$, more than $30 \mathrm{~mm}$ ).

\section{Immunohistochemistry}

For immunohistochemical analysis, of 303 patients, 50 HCC patients (24 patients with HCV, 9 with HBV, 10 with both viruses, and 7 with unknown etiology; 5 patients with well-, 3 with well $\sim$ moderately-, 32 with moderately-, 3 with moderately poorly-, and 7 with poorly-differentiated $\mathrm{HCC}$ ) with 35 positive and 15 negative conventional tumor markers, who underwent surgical treatment, were chosen. The immunohistochemical procedures were done as reported previously [25]. The sections were incu- 


\begin{tabular}{|c|c|c|c|c|c|c|}
\hline Clinical parameters & \multicolumn{2}{|c|}{$\begin{array}{c}\text { No. of HCC patient } \\
303\end{array}$} & $\frac{\text { hTERT mRNA }}{p}$ & $\frac{\text { AFP }}{p}$ & $\frac{\text { AFP-L3 }}{p}$ & $\begin{array}{c}\text { DCP } \\
p\end{array}$ \\
\hline \multicolumn{3}{|c|}{ Age mean:65 years old (range:22 to 101 ) } & NS & NS & NS & NS \\
\hline \multirow[t]{2}{*}{ Gender } & & 196 & NS & NS & NS & NS \\
\hline & & 107 & & & & \\
\hline \multirow[t]{4}{*}{ Etiology } & & 97 & NS & NS & NS & NS \\
\hline & & 167 & & & & \\
\hline & $\mathrm{HCV}$ & 24 & & & & \\
\hline & & 15 & & & & \\
\hline \multirow[t]{2}{*}{ Background lesion } & $\mathrm{CH}$ & 113 & NS & NS & NS & NS \\
\hline & LC & 190 & & & & \\
\hline \multirow[t]{3}{*}{ Numbers of tumors } & 1 & 199 & NS & NS & 0.003 & 0.029 \\
\hline & 2 & 55 & & & & \\
\hline & $>3$ & 49 & & & & \\
\hline \multirow[t]{4}{*}{ Tumor size $(\mathrm{cm})$} & $<1$ & 25 & $<0.001$ & 0.008 & NS & NS \\
\hline & $1 \sim 2$ & 79 & & & & \\
\hline & $2 \sim 3$ & 100 & & & & \\
\hline & $>3$ & 99 & & & & \\
\hline \multicolumn{3}{|c|}{ Differentiation of tumor } & $<0.001$ & 0.019 & 0.001 & NS \\
\hline \multirow[t]{5}{*}{ (Edmondson grade) } & $I$ & 24 & & & & \\
\hline & II & 43 & & & & \\
\hline & III & 33 & & & & \\
\hline & IV & 1 & & & & \\
\hline & nown & 101 & & & & \\
\hline
\end{tabular}

hTERT mRNA was independently correlated with tumor size eand differentiation degree. NS: not significant.

Figure 1 Multivariate analysis of tumor markers with clinical parameters in patients with HCC

bated with the following monoclonal antibodies: antihTERT (Santa Cruz Biotechnology, Santa Cruz, CA, USA), anti-Ki67 (Santa Cruz Biotechnology), antiTUNEL (Sigma Chemical, MO, USA), HBsAg (Sigma Chemical), and HCV core antibody (Sigma Chemical). Expression degree was confirmed and estimated of hTERT, Ki-67, and TUNEL by the percentage of positively-stained cell number [26-28].

\section{Statistical analysis}

Multivariate analysis was performed using SPSS 13.0 (SPSS Corp., Tokyo, Japan). Stratified categories in each clinical parameter were evaluated by One Way ANOVA and multivariate analysis using a logistic regression analysis model. To assess the accuracy of the diagnostic tests, the matched data sets (chronic liver diseases patients and HCC patients) regarding AFP, AFP-L3, DCP, and hTERTmRNA were analyzed by using receiver operator characteristic (ROC) curve analysis. The correlation of hTERTmRNA between HCC tissue and serum was analyzed using both Paired t test and Spearman's test. The detection rates of $\mathrm{HCC}$ in comparison with tumor size were evaluated by Friedman test.

\section{Results}

RNA extraction and Real-time quantitative RT-PCR

In each quantitative assay, a strong linear relation was demonstrated between copy number and PCR cycles using RNA controls for concentration $\left(\mathrm{r}^{2}>0.99\right.$; data not shown). hTERTmRNA expression showed stepwise upregulation with disease progression and the quantification was significantly higher in $\mathrm{HCC}$ than in $\mathrm{LC}, \mathrm{CH}$ and healthy individuals $(\mathrm{P}<0.001, \mathrm{P}<0.01$ and $\mathrm{P}<0.001$, respectively, Figure 2A). ROC curve analyses showed that the sensitivity/specificity of hTERTmRNA for HCC were 90.2\%/85.4\% (Figure 2B). Optimal cut-off values for hTERTmRNA expressions were predicted as 9,332 copies $/ 0.2 \mathrm{ml}$ by stressing the higher specificity. Forty six (15\%) of HCC patients, whose AFP, AFP-L3, and DCP were within normal limits, had $4.23 \pm 0.32$ logarithmic values of hTERTmRNA, and 20 patients of 46 patients were positive for this assay.

Multivariate analysis showed that hTERTmRNA was associated with tumor size and differentiation degree of tumor $(\mathrm{P}<0.001$, each, Figure $1 \& 3)$. However, hTERTmRNA was not associated with age, gender, etiology, background lesion or number of tumor. On the other hand, AFP was related to tumor size and differentiation 


\section{A. hTERT mRNA level in liver diseases}

\section{B. ROC curve analysis}
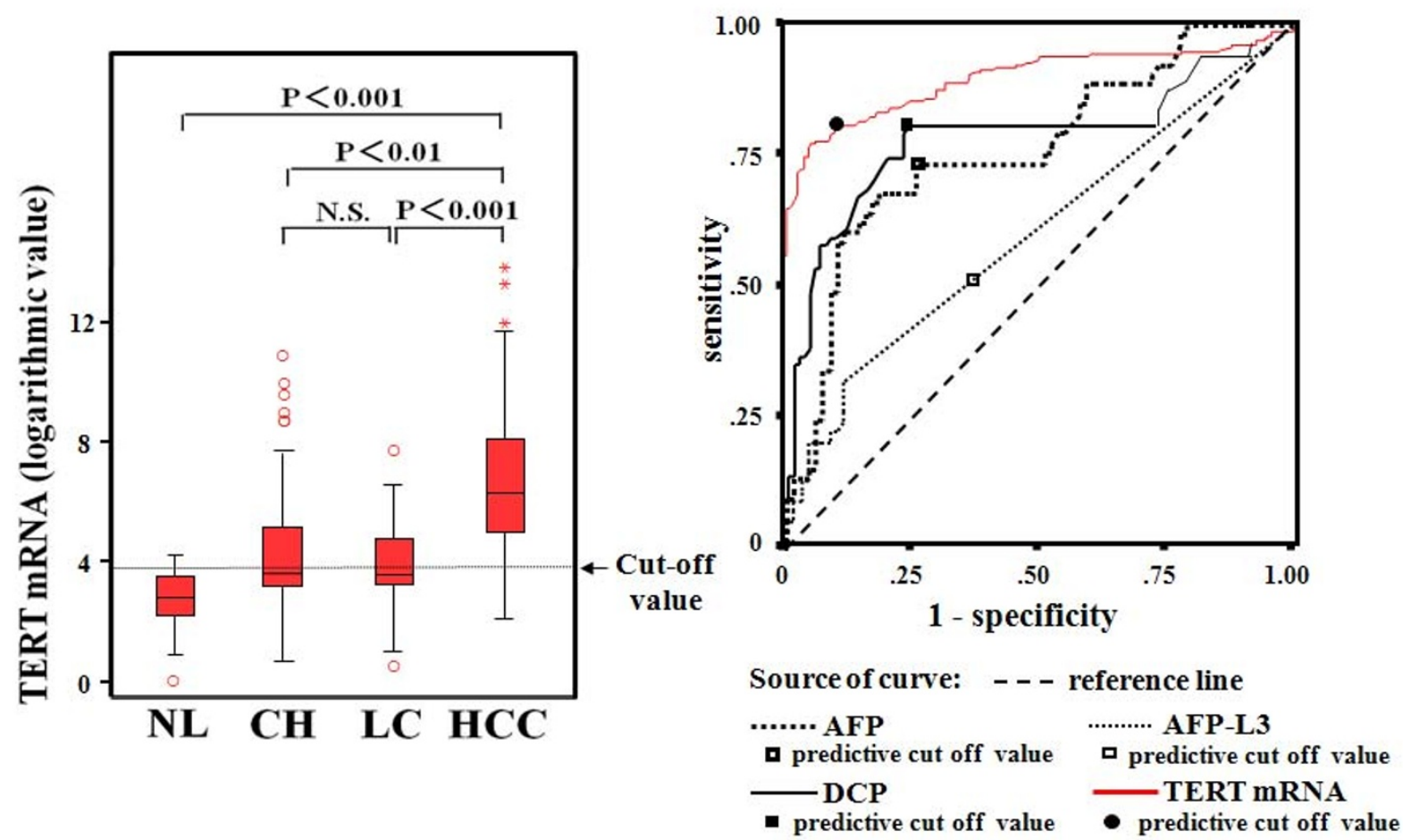

Figure 2 Serum hTERTmRNA and AFP mRNA expression in patients with $\mathrm{HCC}$, LC, and CH, and in healthy individuals and receiver operator characteristics (ROC) curve analyses of hTERTmRNA, AFP, AFP-L3, and DCP. A. hTERTmRNA level in liver diseases. Serum hTERTmRNA levels and AFPmRNA level in patients with HCC, LC, CH, and healthy individuals by real-time RT-PCR were shown. The $95 \%$ confidence interval in each group is shown beside the dots. Significant differences between 4 groups are shown in the upper part of the figure. NL, individual with normal liver; $\mathrm{CH}$, chronic hepatitis; LC, liver cirrhosis; HCC, hepatocellular carcinoma. Optimal cut-off values for hTERTmRNA expressions were predicted as 9,332 copies/0.2 ml. B. ROC curve analysis of hTERTMRNA, AFP, AFP- L3, and DCP was obtained by importing quantified raw data into SPSS II software. The data were analyzed by Paired t test $(P=0.01)$ and non-parametric Spearman's test $(P=0.017)$. AUC of each biomarker in ROC curve analysis is shown. Receiver operator characteristic (ROC) curve analysis of hTERTMRNA, AFP, AFP-L3, and DCP was obtained by importing quantified raw data into SPSS II. The solid line, bold dotted line, dotted line, and bold solid line correspond to DCP, AFP, AFP-L3 and hTERTmRNA, respectively.

( $\mathrm{P}=0.008$ and $\mathrm{P}=0.0199)$, AFP-L3 was related to number of tumor and differentiation degree $(\mathrm{P}=0.003$ and $\mathrm{P}$ $=0.001)$, and DCP was associated with only number of tumor $(\mathrm{P}=0.029)$. By Pearson relative test, serum hTERTmRNA significantly associated with tumor size and number of tumors $(\mathrm{P}<0.033$ and $\mathrm{P}<0.003$, respectively, Table 1). Importantly, hTERTmRNA was related only to DCP $(\mathrm{P}=0.03)$.

ROC curve analyses showed that the sensitivity/specificity of hTERTmRNA for HCC were $90.2 \% / 85.4 \%$ (Table 2 ). The sensitivity/specificity of AFP, AFP-L3, and DCP were $76.6 / 66.2,60.5 / 88.7$, and $83.4 / 80.3$, respectively. Thus, hTERTmRNA was superior to other markers especially in sensitivity. The positive predictive value (PPV)/ negative predictive value (NPV) of hTERTmRNA were 83.0/85.9. On the other hand, the PPV/NPV for AFP, AFP-L3, and DCP were 74.6/67.7, 59.6/92.2, and 78.4/ 73.5 , respectively. Consequently, hTERTmRNA was superior to other markers in the diagnosis of HCC. Combina- tions of hTERTmRNA with AFP level improved the sensitivity/specificity up to $96.0 \% / 87.2 \%$. ROC curve analysis categorized by viruses was examined and sensitivity/specificity in $\mathrm{HBV}$-infected cases was similar to that of HCV-infected cases (additional file 1). hTERT and other markers in LC was not statistically and significantly different in comparison with that in $\mathrm{CH}$.

\section{Estimation of therapeutic effect and the possibility of early} HCC detection of hTERTmRNA in comparison with other biomarkers

To examine the significance of hTERTmRNA before and after TAE, serum hTERTmRNA was measured before and 7 days after TAE in 16 HCC patients (Figure 4A). As a result, hTERTmRNA significantly decreased after TAE (P $=0.018$ ), suggesting that changes in hTERTmRNA are indicative of therapeutic effects on HCC. Comparing the follow-up data of hTERTmRNA and AFP (Figure 4B, C), 


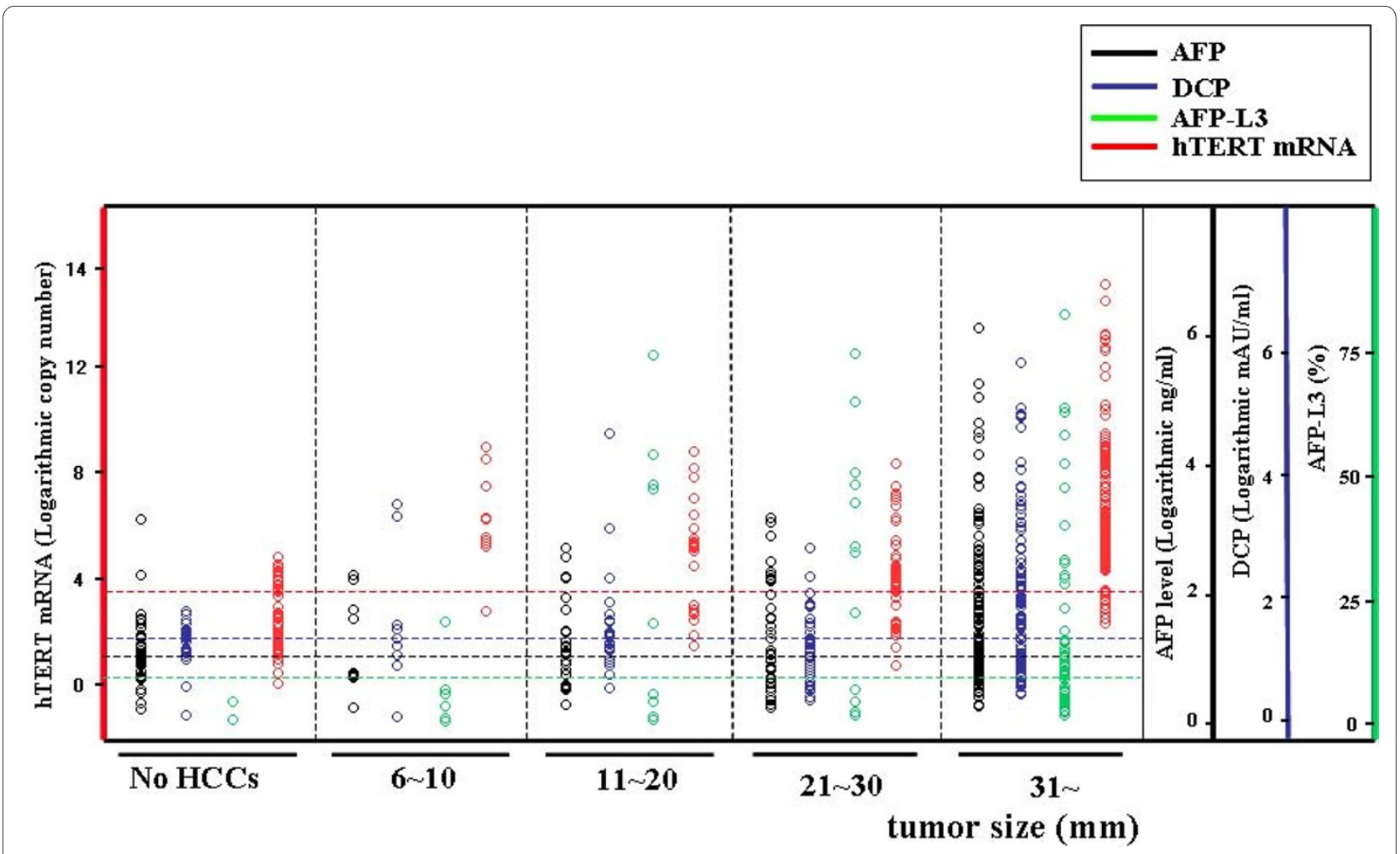

Figure 3 Levels of hTERTmRNA in regard to tumor size $(6-10 \mathrm{~mm}, 11-20 \mathrm{~mm}, 21-30 \mathrm{~mm}$, and over $31 \mathrm{~mm})$.

the half-life of hTERTmRNA was shorter than that of AFP.

To clarify the significance of hTERTmRNA in monitoring the effect of therapies in comparison with other biomarkers, two representative cases were depicted in Figure 5. The quantification of hTERTmRNA was performed before, 2 and 5 months after RFA in a 73-year-old male patient whose $\mathrm{HCC}$ was a single $21 \mathrm{~mm}$-sized (Figure 5A). hTERTmRNA changed similar to AFP, AFP-L3, and DCP, suggesting that hTERTmRNA is useful for monitoring the clinical course of HCC. In a 78-year-old female patient whose $\mathrm{HCC}$ was a single $38 \mathrm{~mm}$-sized, a surgical operation was performed (Figure 5B). The values of AFP, DCP, and hTERTmRNA were measured before, 2 and 7 months after the operation. The operation was performed successfully in this patient, however recurrence was found by dynamic $\mathrm{CT}$ at 7 months after the operation. Although neither AFP nor DCP detected the recurrence, only hTERTmRNA did. In all the cases that hTERT detected recurrence in the earlier stage, no other imaging modality could detect it at the same time, but when we could find HCC in images such as US, CT, or MR, other markers began to arise.

Finally, we examined the relationship between the positive rates of biomarkers and tumor size. Positive rate of hTERTmRNA was higher than that of the other markers in each category of tumor size; 6-10 mm, 11-20 mm, 21$30 \mathrm{~mm}$, over $31 \mathrm{~mm}$ by Friedman test $(\mathrm{P}=0.017)$ (Figure 3). However, the positivity of hTERTmRNA expression tended to reduce slightly in tumors with diameters that exceeded than $51 \mathrm{~mm}(5.2 \pm 1.9$ for 56 patients with 31$50 \mathrm{~mm}$ of HCC, $5.0 \pm 1.8$ for 43 patients with HCC over $51 \mathrm{~mm}$; mean \pm S.D.) (additional file 2). Dot blot regarding the correlation of hTERT mRNA quantification with tumor differentiation is shown in additional file 3. In a 6 mm HCC case, no marker other than hTERTmRNA was elevated and only abdominal US caught the evidence of HCC (Figure 6(a) A, B).

\section{Immunohistochemistry}

Immunohistochemical analysis showed that $\mathrm{Ki}-67$ positivity was observed in the nuclei of cancer cells (Figure 6(b) A). hTERT was observed in both the nuclei and cytoplasm of cancer cells (Figure 6(b) B). Some TUNEL-positive cells were present in cancerous lesions, however the prevalence was low (Figure 6(b) C). hTERT expression was significantly associated with the labeling index of Ki$67(\mathrm{P}=0.023)$. When the labeling indices of Ki-67, hTERT and TUNEL were compared with the differentiation degree of HCC, both hTERT and Ki-67 were higher in poorly differentiated $\mathrm{HCC}$ than in well and moderately differentiated HCC (Figure 6(b) D). 
Table 1: The sensitivity/specificity of each tumor marker for hepatocellular carcinoma and a statistic evaluation of hTERTmRNA level to clinical parameter were shown.

\begin{tabular}{|c|c|c|c|}
\hline clinical parameter & average \pm S.E. & $\begin{array}{l}\text { Pearson test } \\
\text { P value }\end{array}$ & $\begin{array}{c}\text { Multivariate analysis } \\
\quad \mathrm{P} \text { value }\end{array}$ \\
\hline tumor size (mm) & $21.2 \pm 0.1$ & 0.033 & $<0.001$ \\
\hline \multicolumn{4}{|l|}{ (range: 6-90) } \\
\hline tumor number & $1.8 \pm 0.1$ & 0.003 & N.S. \\
\hline tumor differentiation & & N.S. & $<0.001$ \\
\hline $\operatorname{AFP}(\mathrm{ng} / \mathrm{ml})$ & $6146 \pm 4554$ & N.S. & N.S. \\
\hline \multicolumn{4}{|l|}{$(n=353)$} \\
\hline AFP-L3 (\%) & $6.7 \pm 1.0$ & N.S. & N.S. \\
\hline \multicolumn{4}{|l|}{$(n=213)$} \\
\hline \multirow[t]{2}{*}{$\mathrm{DCP}(\mathrm{mAU} / \mathrm{ml})$} & $18780 \pm 1044$ & 0.03 & N.S. \\
\hline & $n=346)$ & & \\
\hline
\end{tabular}

\section{Discussion}

Since HCC has been recently classified as a complex disease with a wide range of risk factors and many cellular signaling pathways have been reported to be involved in hepatocarcinogenesis, a novel biomarker for HCC is required [21]. Since an epoch-making assay to detect telomerase activity was established [11], telomerase has been examined in many kinds of cancers, precancerous lesions and normal tissues using the telomeric repeat amplification protocol and investigated the correlation with telomere length $[29,30]$. Notwithstanding that telomerase was definitely an unprecedented candidate tumor marker due to its specificity to cancer, it has clinically remained inapplicable because telomerase expres-

Table 2: The sensitivity/specificity of each tumor marker for HCC was depicted.

\begin{tabular}{|c|c|c|c|c|c|}
\hline & Sensitivity & Specificity & OR & PPV/NPV & Cut-off point \\
\hline hTERTMRNA & 90.2 & 85.4 & 19.0 & $83.0 / 85.9$ & $\begin{array}{c}\text { 3. } 97 \text { (logarithmic } \\
\text { copy number) }\end{array}$ \\
\hline AFP & 76.6 & 66.2 & 11.1 & 74.6/67.7 & $<10(\mathrm{ng} / \mathrm{ml})$ \\
\hline AFP-L3 & 60.5 & 88.7 & 2.2 & $59.6 / 92.2$ & $<10(\%)$ \\
\hline DCP & 83.4 & 80.3 & 7.6 & $78.4 / 73.5$ & $<40(\mathrm{mAU} / \mathrm{ml})$ \\
\hline
\end{tabular}

The sensitivity/specificity values are $90.2 \% / 85.4 \%$ for hTERTmRNA, $76.6 \% / 66.2 \%$ for AFP, $60.5 \% / 88.7 \%$ for AFP-L3, and 83.4\%/80.3\% for DCP. Regarding a diagnostic assessment in sensitivity and specificity, hTERTmRNA is identified as the most excellent tumor marker. OR: odds ratio, PPV: positive predictive value (\%), NPV: negative predictive value (\%). 


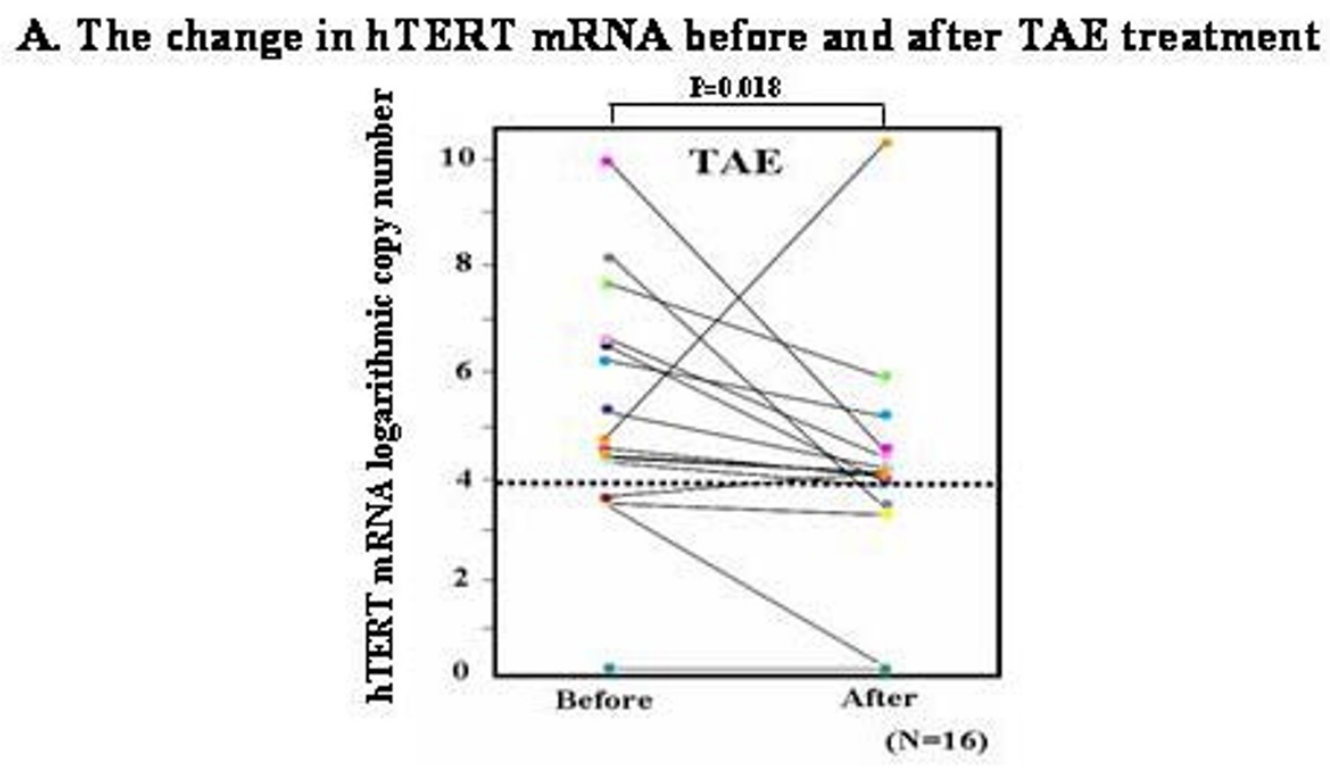

B. Follow-up of hTERT mRNA after TAE C. Follow-up of AFP after TAE
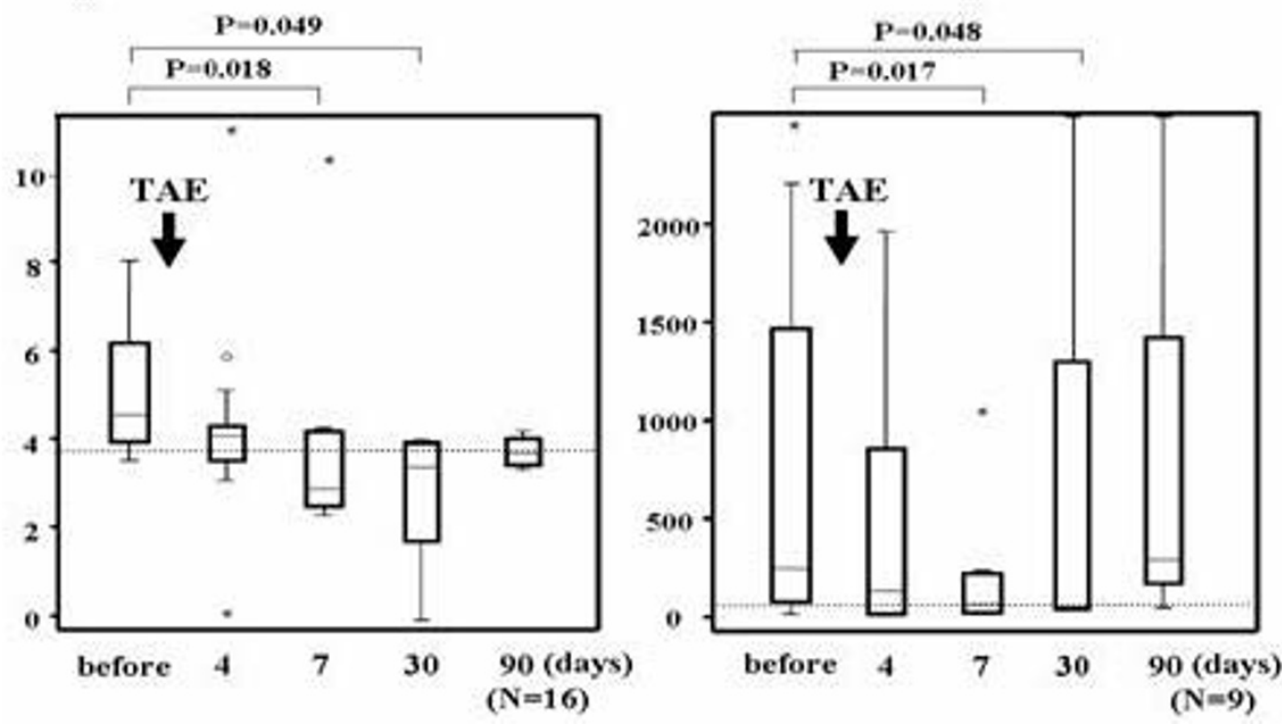

Figure 4 The change of hTERTmRNA before and 7days after TAE. A. Follow-up of serum hTERTmRNA before, 4, 7, 30 and 90 days after TAE. B. Follow-up of serum AFP before, 4, 7, 30 and 90 days after TAE.

sion has not been detected stably in body fluid [12]. In serum, the hTERTmRNA derived from cancer cells seemed to be undetectable because it becomes instable by RNase in blood. Since RNAs in serum are unexpectedly stable within $24 \mathrm{hrs}$ after drawing blood due to particle-associated complex in structure $[13,14]$, it has been suggested that they can be generally detected even in RNase-rich blood. Actually, hTERTmRNA can be detected in serum from breast cancer patients and its maximum sensitivity and specificity are at most $40 \%$ and $100 \%$, respectively [4]. The sensitivity in patients with HCC rose to $89.7 \%$ in the semi-quantitative assay, and thus compared favorably with the previous findings in which the sensitivity and specificity of AFPmRNA were $69 \%$ and $50 \%$ for HCC, respectively [31]. Besides, with respect to $\mathrm{HCC}$ detection, AFPmRNA was superior to AFP level used routinely in clinic [32]. Recently, in the present study, we reported the sensitivity to detect the nucleotides in blood in the process of RNA extraction, including centrifugation steps less than $1500 \times \mathrm{g}$ to remove cellular proteins in serum and a primer set that can detect hTERTmRNA more efficiently than primers in the previous reports (data not shown). We previously reported that hTERT expression was very faint in the 


\section{A. RFA-treated case (male, 73 years ald); $21 \mathrm{~mm}$, single nadule}

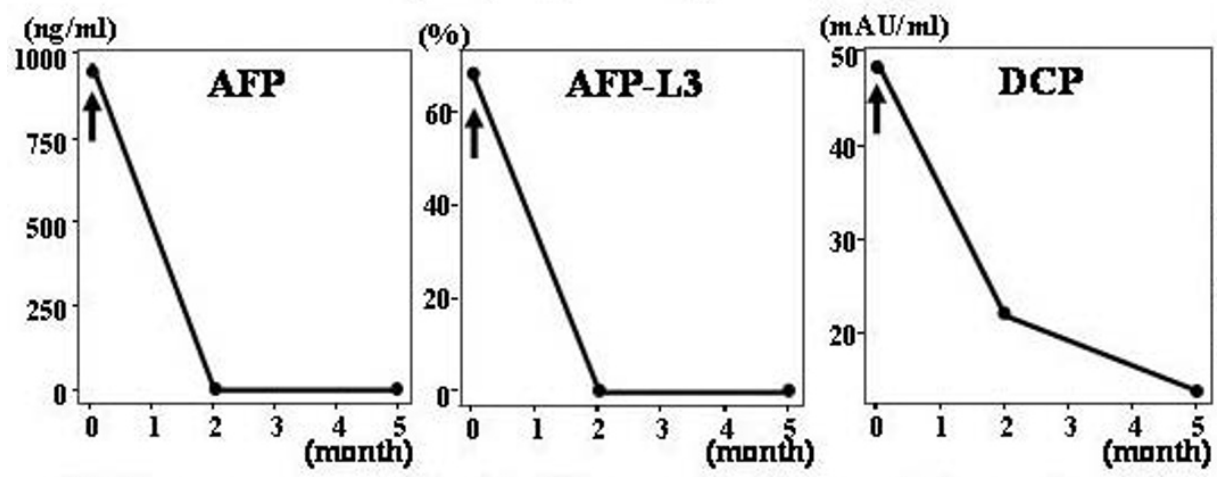

(Logarithuic copy number)

B. A recurrence case (female, 78 years old) after surgical operation, $38 \mathrm{~mm}$ of single HCC (ng/ml) (mAL/ml)
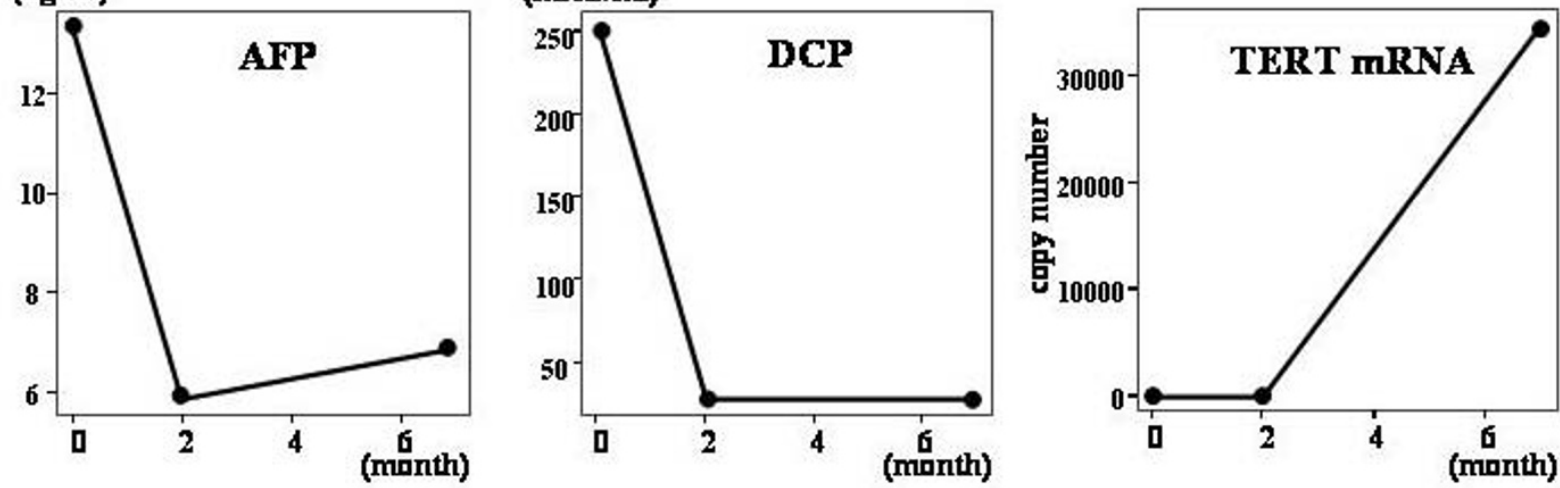

Figure 5 Conventional tumor markers and hTERTmRNA detection during clinical course with therapeutic modalities. A. Changes of AFP, AFPL3, DCP and hTERTmRNA in RFA-treated case (male, 73 years old), of which HCC was $21 \mathrm{~mm}$-sized and single. B.Changes of AFP, DCP and hTERTmRNA in surgical operation-treated case (female, 78 years old), of which HCC was $38 \mathrm{~mm}$-sized and single.

serum from normal individuals indicating that lymphocytes and circulating normal cells express very low levels of hTERTmRNA [9]. Because hTERTmRNA in lymphocytes is very low, elevated hTERTmRNA levels in serum may mean that hTERTmRNA is derived from cancer cells. Since we could detect negligible amounts of lymphocyte markers after three steps of centrifugation of blood samples, the RNA extraction procedure seemed to remove lymphocytes effectively. In addition, normal or damaged hepatocytes express negligible amounts of hTERT [33,34]. Furthermore, we previously showed the significant correlation of hTERTmRNA expression between tumor tissue and serum [32]. These data suggest that hTERTmRNA detected in serum is derived from tumor cells.

Previously, we reported that qualitative analysis of serum hTERTmRNA was superior to AFP for the purpose of the early detection of HCC, because hTERTmRNA was detectable in HCC patients with normal AFP levels [9]. AFP is being widely used as a reliable marker of HCC not in earlier stage but in the advanced stage [35]. However, in this study, neither AFP was able to distinguish HCC from non-cancerous liver diseases, nor hTERTmRNA was correlated with AFP level $(\mathrm{P}=0.201)$, suggesting that quantitative analysis of serum hTERTmRNA was much more sensitive for HCC diagnosis even in the early stage. Because the induction of the abdominal (enhanced-)US, $\mathrm{CT}$, and MRI into the clinical scene enabled us to detect smaller-sized HCC [36], the sensitivity of AFP in the early detection of HCC became less than 70\%. Unlike AFP level, AFPmRNA was significantly correlated with hTERTmRNA $(\mathrm{P}<0.001)$ and more sensitive than AFP. In the present study, we measured AFP-L3, since AFP-L3 has been reported to be a more $\mathrm{HCC}$-specific marker than AFP [37]. Indeed, the level of AFP-L3 correlated significantly with differentiation and number of HCC although that of AFP was correlated with tumor size and differentiation.

In the present study, of $303 \mathrm{HCC}$ patients, 24 patients were negative below the calculated cut-off value $(9,332$; 3.97 as logarithmic number) for serum hTERTmRNA. Although the reason why hTERTmRNA was negative in 
(a)
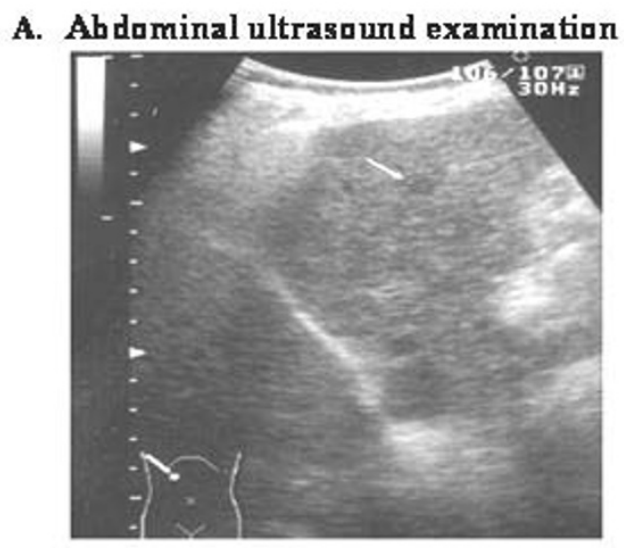

B. Abdominal computed tomography

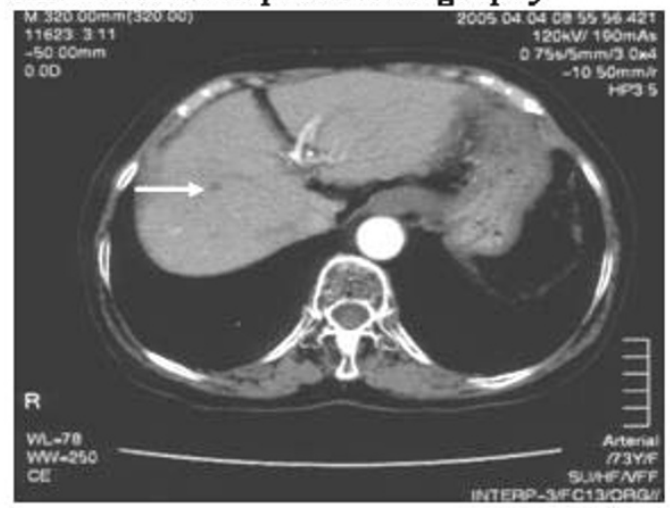

D. (b)

\section{A. $\mathrm{Ki}-67$}

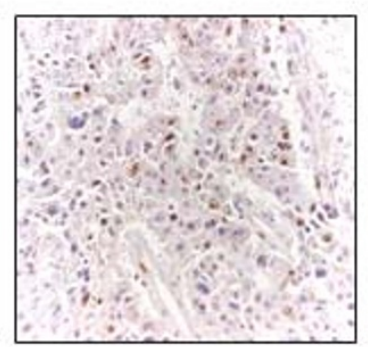

B. hTERT

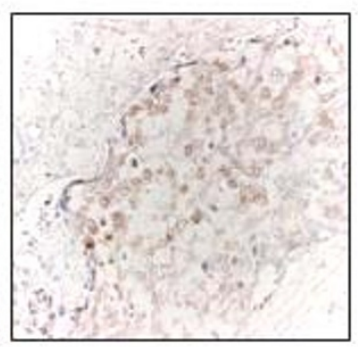

C. TUNNEL

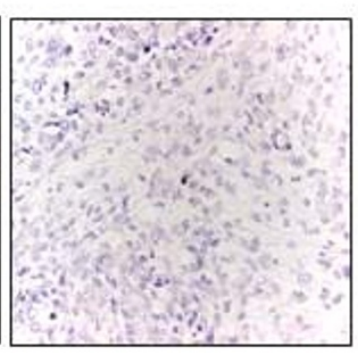

(\%)

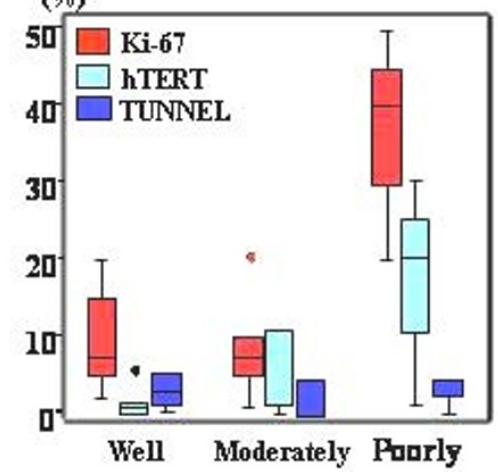

Figure 6 Early detection of small HCC by circulating hTERT mRNA and immunohistochemical analysis of HCC tissues. (a) Imaging diagnosis regarding case with $6 \mathrm{~mm} \mathrm{HCC}$. A. Ultrasonography and B. computed tomography of the smallest HCC detected by hTERTmRNA. The diameter of HCC was 6 mm. Left, ultrasonography; right, CT (b) Immunohistochemical analysis of HCC tissues. A. Ki-67 staining ( $\times 400)$, B. hTERT staining ( $\times 400)$, C. TUNEL ( $\times 400)$, D. Labeling indices of Ki-67, hTERT and TUNEL in regard to differential degree of HCC.

these patients is not clear, eleven of 24 hTERTmRNAnegative HCC patients had decompensated liver cirrhosis as the underlying disease. It has been reported that decompensated liver cirrhosis had higher levels of serum TGF- $\beta$ that promotes apoptosis of immortalized hepatocytes and, in these cases, elevated TGF- $\beta$ may stimulate apoptosis, resulting in reduction of hTERTmRNA $[34,38,39]$. hTERT-negative cases had no other common characteristics with age, gender, etiology, child classification etc. than tumor size, ALT, and surrounding lesion. In 23 cases (95.8\%), ALT was within 1.5 fold normal limits. In 17 cases (70.8\%), surrounding lesion was LC including decompensated situation. Tumor size in 12 cases (50\%) was over $30 \mathrm{~mm}$, reflecting on the biological features of cancer itself, as referred in Norton-Simon models regard tumor growth [40]. AFP and DCP were positive in 16 $(66.7 \%)$ and 11 (45.8\%) cases, respectively, suggesting that combinative use of these markers contributes to improve the diagnostic specificity.
Thus, hTERTmRNA is not only improved in both sensitivity and specificity but has a close correlation with tumor size and number in an early stage of HCC. Since $\mathrm{HCC}$ repeatedly recurs polyclonally after any treatment as a biological characteristic, the measurement of serum hTERTmRNA makes it possible to recognize recurrence or therapeutic effect in details as well as the usefulness for one-point diagnosis. In this respect, we have to undergo follow-up study after the treatment of HCC [24]. hTERTmRNA expression was closely associated with well to moderate differentiation degree of HCC and was enhanced with the proliferation. We should clarify that serum hTERTmRNA can be detected by what alterations of other molecules during the cancer progression [41-43]. In lower differentiated HCC, tumor cells are proliferating and hTERTmRNA has a tendency to correlate with the differentiation degree and an apoptotic event never reflect on the serum detection of cancer cell-derived mRNAs (Figure 6). Nakashio et al. previously reported the significant correlation of HCC differentiation with 
telomerase expression [44]. The results in the present study confirmed their findings. hTERTmRNA showed more sensitivity and specificity compared with AFPmRNA in HCC patients. However, in liver diseases other than HCC, hTERTmRNA was not correlated with AFPmRNA. The higher specificity of hTERTmRNA in HCC may be related to fact that AFPmRNA is produced in HCC cells and injured hepatocytes and hTERT is produced mainly in HCC cells. Furthermore, we could detect serum hTERTmRNA expression even in HCC patients with less than $10 \mathrm{~mm}$ moderate-differentiated tumor, indicating that hTERT are upregulated during rapid proliferation of tumor at the early phase of oncogenesis, dedifferentiation.

Waguri et al. proved that there exist circulating cancer cells derived from original HCC tissues in blood and they can detect hTERTmRNA in blood [45]. The present study suggests that quantification of hTERTmRNAs in serum has diagnostic implications for HCC. Unless apoptosis of cancer cells contributes to the early detection of HCC using serum mRNA, the essence may be immunoreactions [46]. The development of micro vessels may be also involved in the step [47]. We will evaluate the correlation of prognosis with hTERTmRNA and the availability of hTERTmRNA in other cancers by comparison of hTERTmRNA with other tumor markers [48], and will study its usefulness for inflammatory diseases in which cellular reactions are active [49]. This method depends on RNA stability in each process of RNA purification, storage, and quantification. In the light of its superior positivity to other markers, the assay will be applied for clinical use in the strict condition because it is required to keep the serum RNA as it is in blood and avoid the degradation of RNA quality. Now we are improving RNA stability and PCR condition to better cost/benefit of this assay. In the future, another large-scale study will be required to confirm our results for monitoring $\mathrm{HCC}$ and the feasibility for its detection even on a primary care level.

\section{Conclusions}

In sum, our results support the suggestion that quantification of circulating hTERTmRNA expression is clinically useful for the early detection of HCC. Furthermore, hTERTmRNA is superior to conventional tumor markers in the diagnosis and recurrence of $\mathrm{HCC}$ at the early stage.

\section{Additional material}

Additional file 1 TIF ROC curve analysis and AUC in measurement categorized by viruses. ROC curve analysis and AUC in measurement categorized by viruses are demonstrated. Sensitivity/specificity of hTERTmRNA expression in HBV-infected cases is similar to that in HCV-infected cases.

Additional file $\mathbf{2}$ MS word Positivity of each marker for HCC. Positivity of each marker for HCC was shown, categorized by tumor size.
Additional file 3 TIF Dot blot regarding the correlation of hTERTmRNA quantification with tumor differentiation. Serum hTERTmRNA quantification in HCC patients $(n=101)$ diagnosed by liver biopsy was shown, categorized by tumor differentiation. The quantification in serum of HCC patients with well-/moderately-/poorly-/un-differentiation was $4.4 \pm$ 1.4/5.4 \pm 2.0/6.3 $\pm 3.3 / 5.9 \pm 1.8($ mean $\pm S D)$.

\section{Abbreviations}

(h)TERT: (human) telomerase reverse transcriptase protein; HCC: hepatocellular carcinoma; HCV: hepatitis C virus; HBV: hepatitis B virus; LC: liver cirrhosis; $\mathrm{CH}$ : chronic hepatitis; AFP: a-fetoprotein; DCP: des- $\gamma$-carboxy prothrombin; ALT: alanine aminotransferase; Alb: albumin; CNA: Circulating nucleic acids.

\section{Competing interests}

The authors declare that they have no competing interests.

\section{Authors' contributions}

YO analyzed biomedical data and provided blood sample as main researcher in Osaka Red Cross Hospital. MN analyzed biomedical data in Kinki University. MK analyzed biomedical data and provided blood sample as main researcher in Kinki University. KY analyzed biomedical data and provided blood sample in San-in Labor Welfare Hospital. TK analyzed clinical data and the practical analysis in San-in Labor Welfare Hospital. KO analyzed HCC imaging data and the analysis in Saiseikai Gotsu General Hospital. YK was in charge for case study in Saiseikai Gotsu General Hospital. SM analyzed biomedical data and provided blood sample as main researcher in Saiseikai Gotsu General Hospital EN was in charge for case study and biomedical analysis in Saiseikai Gotsu General Hospital. YH analyzed clinical and biomedical data as main researcher in Saiseikai Gotsu General Hospital comprehensively. MK analyzed biomedical data and provided blood sample as main researcher in Matsue City Hospital. SS analyzed biomedical data and provided blood sample as main researcher in Fukuoka University Chikushi Hospital. YH performed biomedical and clinical analysis in surgical case in Tottori University. HK analyzed biomedical data and provided blood sample as chief researcher in San-in Labor Welfare Hospital. JH provided the environment to analyze the data comprehensively. All authors read and approved the final manuscript.

\section{Acknowledgements}

This study was supported by a Grant-in-Aid (18390208) for scientific research from the Ministry of Education, Science, and Culture and the Foundation for the Promotion of Cancer Research in Japan. All the PCR primers were designed by INTEC Web and Genome Informatics, Corporation (Tokyo, Japan).

\section{Author Details}

1Division of Pharmacotherapeutics, Department of Pathophysiological and Therapeutic Science, Faculty of Medicine, Tottori University, 86 Nishicho, Yonago, Tottori 683-8503, Japan, 2Department of Gastroenterology, Osaka Red Cross Hospital, 5-30 Fudegasaki-cho, Tennouji-ku, Osaka, Osaka 543-8555, Japan, ${ }^{3}$ Department of gastroenterology, Kinki University, 3-4-1 Kowakae, Higashi-Osaka, Osaka 577-8502, Japan, 4 Department of Gastroenterology Matsue City Hospital, 32-1 Noshira-cho, Matsue, Shimane 690-8509, Japan, 5Department of Internal Medicine, Shimaneken Saiseikai Gotsu General Hospital, 1551 Gotsu-cho, Gotsu, Shimane 695-8505, Japan, ${ }^{6}$ Division of Organ Pathology, Faculty of Medicine, Tottori University, Nishicho 86, Yonago, 6838503, Japan, ${ }^{7}$ Internal Medicine, San-in Labor Welfare Hospital, 1-8-1 Kaikeshinden, Yonago, Tottori 683-0002, Japan, 8Department of Gastroenterology, Fukuoka University Chikushi Hospital, 1-1-1 Zokumyoin, Chikusino, Fukuoka 818-8502, Japan, 9Department of Pathobiological Science and Technology, School of Health Science, Faculty of Medicine, Tottori University, 86 Nishicho, Yonago, Tottori 683-8503, Japan and 10Division of Molecular and Genetic Medicine, Department of Genetic Medicine and Regenerative Therapeutics, Tottori University School of Medicine, 86 Nishicho, Yonago, Tottori 683-8503, Japan

Received: 23 July 2009 Accepted: 18 May 2010

Published: 18 May 2010

\section{References}

1. Moyzis RK, Buckingham JM, Cram LS, Dani M, Deaven LL, Jones MD, Meyne J, Ratliff RL, Wu JR: A highly conserved repetitive DNA sequence, 
(TTAGGG)n, present at the telomeres of human chromosomes. Proc Natl Acad Sci USA 1988, 85:6622-6626.

2. Paradis V, Dargère $D$, Laurendeau I, Benoît $G$, Vidaud M, Jardin A, Bedossa $P$ : Expression of the RNA component of human telomerase (hTR) in prostate cancer, prostatic intraepithelial neoplasia, and normal prostate tissue. J Pathol 1999, 189:213-218.

3. Kopreski MS, Benko FA, Kwak LW, Gocke CD: Detection of tumor messenger RNA in the serum of patients with malignant melanoma. Clin Cancer Res 1999, 5:1961-1965.

4. Chen XQ, Bonnefoi H, Pelte MF, Lyautey J, Lederrey C, Movarekhi S, Schaeffer P, Mulcahy HE, Meyer P, Stroun M, Anker P: Telomerase RNA as a detection marker in the serum of breast cancer patients. Clin Cancer Res 2000, 6:3823-3826.

5. El-Serag HB, Mason AC: Rising incidence of hepatocellular carcinoma in the United States. N Engl J Med 1999, 340:745-750.

6. Shirabe K, Takenaka K, Taketomi A, Kawahara N, Yamamoto K, Shimada M, Sugimachi K: Postoperative hepatitis status as a significant risk factor for recurrence in cirrhotic patients with small hepatocellular carcinoma. Cancer 1996, 15:1050-1055.

7. Dohmen K, Shirahama M, Onohara S, Miyamoto Y, Torii Y, Irie K, Ishibashi $\mathrm{H}$ : Differences in survival based on the type of follow-up for the detection of hepatocellular carcinoma: an analysis of 547 patients. Hepatol Res 2000, 18:110-121.

8. Miura N, Horikawa I, Nishimoto A, Ohmura H, Ito H, Hirohashi S, Shay JW, Oshimura M: Progressive telomere shortening and telomerase reactivation during hepatocellular carcinogenesis. Cancer Genet Cytogenet 1997, 93:56-62.

9. Miura N, Shiota G, Nakagawa T, Maeda Y, Sano A, Marumoto A, Kishimoto Y, Murawaki Y, Hasegawa J: Sensitive detection of hTERTmRNA in the serum of patients with hepatocellular carcinoma. Oncology 2003, 64:430-434

10. Mitas M, Mikhitarian K, Walters C, Baron PL, Elliott BM, Brothers TE, Robison $J G$, Metcalf JS, Palesch YY, Zhang Z, et al:: Quantitative real-time RT-PCR detection of breast cancer micrometastasis using a multigene marker panel. Int J Cancer 2001, 93:162-171.

11. Kim NW, Piatyszek MA, Prowse KR, Harley CB, West MD, Ho PL, Coviello GM, Wright WE, Weinrich SL, Shay JW: Specific association of human telomerase activity with immortal cells and cancer. Science 1994, 266:2011-2015.

12. Tatsuma T, Goto S, Kitano S, Lin YC, Lee CM, Chen CL: Telomerase activity in peripheral blood for diagnosis of hepatoma. J Gastroenterol Hepatol 2000, 15:1064-1070.

13. Ng EK, Tsui NB, Lam NY, Chiu RW, Yu SC, Wong SC, Lo ES, Rainer TH, Johnson PJ, LO YM: Presence of filterable and non filterable mRNA in the plasma of cancer patients and healthy individuals. Clin Chem 2002, 48:1212-1217.

14. Tsui NB, Ng EK, Lo YM: Stability of Endogeneous and added RNA in blood specimens, serum, and plasma. Clin Chem 2002, 48:1647-1653.

15. Nørgaard R, Kassem M, Rattan SI: Heat shock-induced enhancement of osteoblastic differentiation of hTERT-immortalized mesenchymal stem cells. Ann N Y Acad Sci 2006, 1067:443-447.

16. Zhang X, Soda Y, Takahashi K, Bai Y, Mitsuru A, Igura K, Satoh H, Yamaguchi S, Tani K, Tojo A, et al: Successful immortalization of mesenchymal progenitor cells derived from human placenta and the differentiation abilities of immortalized cells. Biochem Biophys Res Commun 2006, 29:853-859.

17. Gabet AS, Accardi R, Bellopede A, Popp S, Boukamp P, Sylla BS, LondoñoVallejo JA, Tommasino M: Impairment of the telomere/telomerase system and genomic instability are associated with keratinocyte immortalization induced by the skin human papillomavirus type 38 . FASEB J 2008, 22:622-632.

18. Gandellini P, Folini M, Bandiera R, De Cesare M, Binda M, Veronese S, Daidone MG, Zunino F, Zaffaroni N: Down-regulation of human telomerase reverse transcriptase through specific activation of RNAi pathway quickly results in cancer cell growth impairment. Biochem Pharmacol 2007, 73:1703-1714

19. Burnworth B, Arendt S, Muffler S, Steinkraus V, Bröcker EB, Birek C, Hartschuh W, Jauch A, Boukamp P: The multi-step process of human skin carcinogenesis: a role for p53, cyclin D1, hTERT, p16, and TSP-1. Eur J Cell Biol 2007, 86:763-780.

20. Xu L, Blackburn EH: Human cancer cells harbor T-stumps, a distinct class of extremely short telomeres. Mol Cell 2007, 26:315-327.
21. Aravalli RN, Steer CJ, Cressman ENK: Molecular mechanisms of hepatocellular carcinoma. Hepatology 2008, 48:2047-2063.

22. Miura N, Nakamura H, Sato R, Tsukamoto T, Harada T, Takahashi S, Adach Y, Shomori K, Sano A, Kishimoto Y, et al:: Clinical usefulness of serum telomerase reverse transcriptase (hTERT) mRNA and epidermal growth factor receptor (EGFR) mRNA as a novel tumor marker for lung cancer. Cancer Sci 2006, 97:1366-1373

23. Miura N, Kanamori Y, Takahashi M, Sato R, Tsukamoto T, Takahashi S, Harada T, Sano A, Shomori K, Harada T, et al:: A diagnostic evaluation of serum human telomerase reverse transcriptase mRNA as a novel tumor marker for gynecologic malignancies. Oncol Rep 2007, 17:541-548

24. Tani N, Ichikawa D, Ikoma D, Tomita H, Sai S, Ikoma H, Fujiwara H, Kikuchi S, Okamoto K, Ochiai T, et al:: Circulating cell-free mRNA in plasma as a tumor marker for patients with primary and recurrent gastric cancer. Anticancer Res 2007, 27:1207-1212.

25. Shomori K, Sakatani T, Goto A, Matsuura T, Kiyonari H, Ito H: Thymidine phosphorylase expression in human colorectal mucosa, adenoma and carcinoma: role of p53 expression. Pathol Int 1999, 49:491-499.

26. Yeh TS, Chen TC, Chen MF: Dedifferentiation of human hepatocellular carcinoma up-regulates telomerase and Ki-67 expression. Arch Surg 2000, 135:1334-1339.

27. Tahara H, Yasui W, Tahara E, Fujimoto J, Ito K, Tamai K, Nakayama J, Ishikawa F, Tahara E, Ide T: Immuno-histochemical detection of human telomerase catalytic component, hTERT, in human colorectal tumor and non-tumor tissue sections. Oncogene 1999, 18:1561-1567.

28. Takeba Y, Sekine S, Kumai T, Matsumoto N, Nakaya S, Tsuzuki Y, Yanagida Y, Nakano H, Asakura T, Ohtsubo T, et al: Irinotecan-induced apoptosis is inhibited by increased P-glycoprotein expression and decreased p53 in human hepatocellular carcinoma cells. Biol Pharm Bull 2007, 30:1400-1406

29. Nakashio R, Kitamoto M, Tahara H, Nakanishi T, Ide T, Kajiyama G: Significance of telomerase activity in the diagnosis of small differentiated hepatocellular carcinoma. Int J Cancer 1997, 22:141-147.

30. Plentz RR, Park YN, Lechel A, Kim H, Nellessen F, Langkopf BH, Wilkens L, Destro A, Fiamengo B, Manns MP, et al.: Telomere shortening and inactivation of cell cycle checkpoints characterize human hepatocarcinogenesis. Hepatology 2007, 45:968-976.

31. Wong Ih-N, Leung T, Ho S, Lau WY, Chan M, Johnson PJ: Semiquantification of circulating hepatocellular carcinoma cells by reverse transcriptase polymerase chain reaction. Br J Cancer 1997, 76:628-633.

32. Miura N, Maeda Y, Kanbe T, Yazama H, Takeda Y, Sato R, Tsukamoto T, Sato E, Marumoto A, Harada T, et al:: Serum human telomerase reverse transcriptase messenger RNA as a novel tumor marker for hepatocellular carcinoma. Clin Cancer Res 2005, 1:3205-3209.

33. Onishi T, Nouso K, Higashi T, Toshikuni N, Nakatsukasa H, Kobayashi Y, Uemura M, Yumoto E, Fujiwara K, Sato S, et al: Cellular distribution of telomerase reverse transcriptase in human hepatocellular carcinoma. J Gastroenterol Hepatol 2003, 18:1168-1174.

34. Wege H, Chui MS, Le HT, Strom SC, Zern MA: In vitro expansion of human hepatocytes is restricted by telomere-dependent replicative aging. Cell Transplant 2003, 12:897-906.

35. Peng SY, Chen WJ, Lai PL, Jeng YM, Sheu JC, Hsu HC: High alphafetoprotein level correlates with high stage, early recurrence and poor prognosis of hepatocellular carcinoma: significance of hepatitis virus infection, age, p53 and beta-catenin mutations. Int J Cancer 2004, 112:44-50.

36. Leoni S, Piscaglia F, Righini R, Bolondi L: Management of small hepatocellular carcinoma. Acta Gastroenterol Belg 2006, 69:230-235.

37. Okuda H, Nakanishi T, Takatsu K, Saito A, Hayashi N, Yamamoto M, Takasaki K, Nakano M: Clinicopathologic features of patients with hepatocellular carcinoma seropositive for a-fetoprotein- $\mathrm{L} 3$ and seronegative for des$\mathrm{g}$-carboxyprothrombin in comparison with those seropositive for desg-carboxy prothrombin alone. J Gastroenterol Hepatol 2002, 17:772-778.

38. Cavin LG, Romieu-Mourez R, Panta GR, Sun J, Factor VM, Thorgeirsson SS, Sonenshein GE, Arsura M: Inhibition of CK2 activity by TGF-beta 1 promotes IkappaB-alpha protein stabilization and apoptosis of immortalized hepatocytes. Hepatology 2003, 38:1540-1551.

39. Prade-Houdellier N, Frébet E, Demur C, Gautier EF, Delhommeau F, Bennaceur-Griscelli AL, Gaudin C, Martinel V, Laurent G, Mansat-De Mas V, et al:: Human telomerase is regulated by erythropoietin and 
transforming growth factor-beta in human erythroid progenitor cells. Leukemia 2007, 21:2304-2310.

40. Heitjan DF: Generalized Norton-Simon models of tumour growth. Stat Med 1991, 10:1075-1088.

41. Yu GR, Kim SH, Park SH, Cui XD, Xu DY, Yu HC, Cho BH, Yeom YI, Kim SS, Kim SB, et al:: Identification of molecular markers for the oncogenic differentiation of hepatocellular carcinoma. Exp Mol Med 2007. 31:641-652

42. Swisher JF, Khatri U, Feldman GM: Annexin A2 is a soluble mediator of macrophage activation. J Leukoc Biol 2007, 82:1174-1184.

43. Lin SY, Elledge SJ: Multiple tumor suppressor pathways negatively regulate telomerase. Cell 2003, 27:881-889.

44. Takahashi S, Kitamoto M, Takaishi H, Aikata H, Kawakami Y, Nakanishi T, Shimamoto F, Tahara E, Tahara H, Ide T, et al:: Expression of telomerase component genes in hepatocellular carcinoma. Eur J Cancer 2000 36:496-502.

45. Waguri N, Suda T, Nomoto M, Kawai H, Mita Y, Kuroiwa T, Igarashi M, Kobayashi M, Fukuhara Y, Aoyagi Y: Sensitive and specific detection of circulating cancer cells in patients with hepatocellular carcinoma; detection of human telomerase reverse transcriptase messenger RNA after immunomagnetic separation. Clin Cancer Res 2003, 9:3004-3011.

46. Mizukoshi E, Nakamoto Y, Marukawa Y, Arai K, Yamashita T, Tsuji H, Kuzushima K, Takiguchi M, Kaneko S: Cytotoxic T cell responses to human telomerase reverse transcriptase in patients with hepatocellular carcinoma. Hepatology 2006, 43:1284-1294.

47. Piao YF, He M, Shi Y, Tang TY: Relationship between microvessel density and telomerase activity in hepatocellular carcinoma. World J Gastroenterol 2004, 15:2147-2149.

48. Fujita Y, Fujikane T, Fujiuchi S, Nishigaki Y, Yamazaki Y, Nagase A, Shimizu $T$, Ohsaki Y, Kikuchi K: The diagnostic and prognostic relevance of human telomerase reverse transcriptase mRNA expression detected in situ in patients with non small cell lung carcinoma. Cancer 2003, 98:1008-1013.

49. Miura N, Kabashima H, Shimizu M, Sato R, Tsukamoto T, Harada T, Takahashi S, Endo R, Nakayama N, Takikawa Y, et al: Clinical impact of serum transforming growth factor-alpha mRNA as a predictive biomarker for the prognosis of fulminant hepatitis. Hepatol Int 2008 2:213-221.

Pre-publication history

The pre-publication history for this paper can be accessed here: http://www.biomedcentral.com/1471-230X/10/46/prepub

doi: 10.1186/1471-230X-10-46

Cite this article as: Miura et al., A novel biomarker TERTMRNA is applicable for early detection of hepatoma BMC Gastroenterology 2010, 10:46

Submit your next manuscript to BioMed Centra and take full advantage of:

- Convenient online submission

- Thorough peer review

- No space constraints or color figure charges

- Immediate publication on acceptance

- Inclusion in PubMed, CAS, Scopus and Google Scholar

- Research which is freely available for redistribution

Submit your manuscript at www.biomedcentral.com/submit
C Biomed Central 